\title{
Protein Kinase A in the Pedunculopontine Tegmental Nucleus of Rat Contributes to Regulation of Rapid Eye Movement Sleep
}

\author{
Subimal Datta ${ }^{1,2,3}$ and Frank Desarnaud ${ }^{1,2}$ \\ ${ }^{1}$ Laboratory of Sleep and Cognitive Neuroscience, Departments of ${ }^{2}$ Psychiatry and ${ }^{3}$ Neurology, Boston University School of Medicine, Boston, \\ Massachusetts 02118
}

Intracellular signaling mechanisms within the pedunculopontine tegmental (PPT) nucleus for the regulation of recovery rapid eye movement (REM) sleep following REM sleep deprivation remain unknown. This study was designed to determine the role of PPT intracellular cAMP-dependent protein kinase A (cAMP-PKA) in the regulation of recovery REM sleep in freely moving rats. The results show that a brief period $(3 \mathrm{~h})$ of selective REM sleep deprivation caused REM sleep rebound associated with increased PKA activity and expression of the PKA catalytic subunit protein (PKA-CU) in the PPT. Local application of a cAMP-PKA-activation-selective inhibitor, $\operatorname{RpCAMPS}(0.55,1.1$, and $2.2 \mathrm{nmol} / 100 \mathrm{nl} ; n=8 \mathrm{rats} /$ group$)$, bilaterally into the PPT, reduced PKA activity and PKA-CU expression in the PPT, and suppressed the recovery REM sleep, in a dose-dependent manner. Regression analyses revealed significant positive relationships between: PPT levels of PKA activity and the total percentages of REM sleep recovery (Rsqr $=0.944 ; n=40$ rats); PPT levels of PKA-CU expression and the total percentages of REM sleep recovery (Rsqr $=0.937 ; n=40$ rats); PPT levels of PKA-CU expression and PKA activity (Rsqr $=0.945 ; n=40$ rats). Collectively, these results provide evidence that activation of intracellular PKA in the PPT contributes to REM sleep recovery following REM sleep deprivation.

\section{Introduction}

Using a variety of experimental techniques, a number of studies in both humans and animals have provided considerable evidence that supports the role of the pedunculopontine tegmental (PPT) nucleus as a critical area of the brainstem for the regulation of REM sleep (Pace-Schott and Hobson, 2002; Garcia-Rill et al., 2001, 2003, 2008; Datta and Maclean, 2007; Lydic and Baghdoyan, 2008). The PPT is situated in the dorsolateral tegmentum and contains a prominent group of cholinergic neurons as well as some noncholinergic neurons, which project widely throughout the brainstem and forebrain (Mesulam et al., 1983; Garcia-Rill, 1991; Jones, 2004; Wang and Morales, 2009). Single cell recording studies have shown that neurons in the PPT discharge at higher rates during REM sleep than during slow-wave sleep (SWS) (El-Mansari et al., 1989; Steriade et al., 1990; Thakkar et al., 1998; Datta and Siwek, 2002). PPT neurons exhibit REM sleep-associated immunoreactivity of phosphorylated cyclic adenosine monophosphate (cAMP) response element-binding protein (pCREB-IR) and c-Fos protein (c-Fos-IR). Of REM sleep-associated pCREB-IR cells, 95\% were cholinergic (Datta et

Received March 26, 2010; revised June 24, 2010; accepted June 29, 2010.

This work was supported by National Institutes of Health Research Grants MH59839 and NS34004. The content is solely the responsibility of the authors and does not necessarily represent the official views of the National Institutes of Health. We thank M. P. Huang, B. W. Macone, and K. Radadia for technical assistance and Dr. J. Allan Hobson, Dr. Elissa H. Patterson, and Dr. Donald F. Siwek for their valuable discussions on this manuscript.

Correspondence should be addressed to Subimal Datta, Boston University School of Medicine, 85 East Newton Street, Suite: M-902, Boston, MA 02118. E-mail: Subimal@bu.edu.

DOI:10.1523/JNEUROSCI.1563-10.2010

Copyright $\odot 2010$ the authors $\quad 0270-6474 / 10 / 3012263-11 \$ 15.00 / 0$ al., 2009). The active role of the PPT in REM sleep regulation is also supported by localized lesion experiments (Webster and Jones, 1988; Shouse and Siegel, 1992; Deurveilher and Hennevin, 2001).

Recent studies have demonstrated that the excitatory neurotransmitter glutamate activated kainate receptors on PPT neurons to induce REM sleep (Datta and Siwek, 1997; Datta, 2002; Datta et al., 2002), whereas the activation of GABA-B receptors suppressed PPT neuronal activity and REM sleep (Ulloor et al., 2004). Other pharmacological studies suggested that kainate and GABA-B receptors in the PPT may involve a cAMP-dependent protein kinase A (cAMP-PKA) signal transduction pathway to regulate the expression of spontaneous REM sleep (Datta and Prutzman, 2005; Bandyopadhya et al., 2006; Datta, 2007). More recently, it was demonstrated that the elimination of PPT cholinergic cells by local application of excitotoxin into the PPT, prevented homeostatic regulation of REM sleep (Deurveilher and Hennevin, 2001). However, little is known about the molecular mechanisms that are involved in the homeostatic regulation of REM sleep. The goal of the present study was to evaluate the hypothesis that the activation of intracellular cAMP-PKA in PPT cholinergic cells is associated with increase in REM sleep caused by REM sleep deprivation. To achieve this goal, we first determined whether the application of RpCAMPS (an inhibitor of cAMP-PKA activation) into the PPT could block homeostatic regulation of REM sleep. Next, we quantified the levels of PKA activity and PKA catalytic subunit (PKA-CU) in the PPT of vehicle control- and RpCAMPS-treated rats. Finally, the relationship between PPT cAMP-PKA activation and increase in REM sleep 
caused by REM sleep deprivation was confirmed by performing regression analyses between levels of PKA activity, PKA-CU expression, and percentages of REM sleep. The results of this study demonstrate that the application of RpCAMPS in the PPT suppressed PKA activity and increase in REM sleep caused by REM sleep deprivation. These results indicate that the increased activation of cAMP-PKA in PPT cholinergic cells is a critical molecular step for the homeostatic regulation of REM sleep.

\section{Materials and Methods}

Subjects and housing. Experiments were performed on 44 adult male Wistar rats (Charles River) weighing between 200 and 300 g. The rats were housed individually at $24^{\circ} \mathrm{C}$ with free access to food and water. Lights were on from 7:00 A.M. to 7:00 P.M. (light cycle) and off from 7:00 P.M. to 7:00 A.M. (dark cycle). Experiments were performed in accordance with the National Institutes of Health Guide for the Care and Use of Laboratory Animals and were approved by the Boston University Animal Care Committee (AN-14084). Additional care was taken to ensure that any potential discomfort was eliminated, and the number of animals used were minimized. To reduce additional stress that might be imposed by experimental handling, animals were gently handled daily for 15-20 min between 09:00 A.M. and 10:00 A.M. This habituation handling began 1 week before surgery and continued up to the experimental recording sessions.

Surgical procedures for electrode and guide tube implantation. All surgical procedures were performed stereotaxically under aseptic conditions. Animals were anesthetized with pentobarbital (40 mg/kg, i.p.; Ovation Pharmaceuticals), placed in the stereotaxic apparatus, and secured using blunt rodent ear bars as described previously (Paxinos and Watson, 1997). The appropriate depth of anesthesia was judged by the absence of palpebral reflexes and the absence of response to tail pinch. Core body temperature was maintained at $37^{\circ} \pm 1^{\circ} \mathrm{C}$ with a thermostatic heating pad and a rectal thermistor probe. The scalp was cleaned and painted with providone iodine. A scalp incision was made, and the skin was retracted. The skull surface was cleaned in preparation for electrode implantation. Following completion of the surgical procedure, animals were administered saline ( 5 cc, s.c.) to prevent dehydration, and ampicillin (50 mg/rat, i.m.; Bristol-Myers Squibb Company) to control any potential postsurgical infection. Potential postoperative pain was controlled with buprenorphine $(0.05 \mathrm{mg} / \mathrm{kg}$, s.c; Ben Venue Laboratories).

To record behavioral states of vigilance, cortical electroencephalogram (EEG), dorsal neck muscle electromyogram (EMG), and hippocampal EEG (to record theta wave) recording electrodes were chronically implanted, as described previously (Datta, 2000, 2002; Datta et al., 2002). In addition, stainless steel guide tubes (26 gauge) with equal length stylettes were stereotaxically implanted bilaterally $2 \mathrm{~mm}$ above the PPT (in relation to stereotaxic "0": anterior, 1.0; lateral, 1.8; horizontal, 3.0) as described previously (Datta et al., 2001, 2002; Datta, 2002). All electrodes and guide tubes were secured to the skull with dental acrylic. Electrodes were crimped to mini-connector pins and brought together in a plastic connector. Immediately after surgery, animals were placed in recovery cages and monitored for successful recovery from anesthesia and surgery. Successful recovery was gauged by the return of normal posture, voluntary movement, and grooming.

Adaptation recording session. After a postsurgical recovery period of 3-7 d, rats were habituated to the experimenter, the sound-attenuating recording cage (electrically shielded: $2.5 \times 1.5 \times 1.5$ feet), and freely moving polygraphic recording (Grass Model 15 Neurodata Amplifier System, Astro-Med) conditions for 7-10 d. All adaptation recording sessions were performed between 9:00 A.M. and 2:00 P.M., when rats are normally sleeping. These $5 \mathrm{~h}$ habituation sessions were also considered to be baseline recording sessions for electrode testing and monitoring daily variations in the percentages of wake/sleep stages. The last day of these adaptation recording sessions was determined when, for 3 consecutive days, the day-to-day variation in the percentage of REM sleep was $<5 \%$ of the total amount of REM sleep. During recovery, habituation, and free moving recording conditions (adaptation recording sessions), all rats were housed under the same $12 \mathrm{~h}$ light/dark cycle with free access to food and water.

Drug and vehicle for microinjections. The drug used in this study was a specific membrane-permeable inhibitor of cAMP-dependent PKA activation, Rp-Adenosine 3',5'-cyclic monophosphothioate triethylamine (RpCAMPS; molecular weight 446.46), purchased from Sigma Chemical Co.. The RpCAMPS was dissolved in $0.9 \%$ saline at three concentrations $(0.55,1.1$, and $2.2 \mathrm{nmol} / 100 \mathrm{nl})$. Saline $(0.9 \%, 100 \mathrm{nl})$ was used for the vehicle control microinjection. Control saline and drug solutions were freshly prepared under sterile conditions immediately before each use. The selection of this drug was based on its selective inhibitory effect of intracellular cAMP-PKA activation (Van Hasstert et al., 1984; Wang et al., 1991; Shi and Bunney, 1992). Commercially available RpCAMPS is suitable for microinjection studies because it is water soluble, cellpermeable, has reversible effects, and has been used successfully for microinjection studies in behaving animals (Cook et al., 1995; Self and Nestler, 1995; Capece and Lydic, 1997; Punch et al., 1997; Self et al., 1998; Schafe and LeDoux, 2000; Bandyopadhya et al., 2006).

Polygraphic recordings and REM sleep deprivation setup. To record cortical EEG, EMG, and hippocampal EEG in freely moving conditions, each head plug was mated to a male connector connected to a commutator. Signals from the commutator went to a polygraph (Grass Model 15 Neurodata Amplifier System, located in the next room) via its electrode board (located inside the recording chamber). To allow rats to move freely inside the recording cage while maintaining the head plug connection, a counterbalanced connecting cable and a mechanical pulley system (attached to the roof of the recording chamber) were used. In a separate room, polygraphic signs of the rat were continuously observed on a computer monitor to identify ongoing behavioral stages (Datta et al., 2004, 2009; Bandyopadhya et al., 2006).

For REM sleep deprivation, the beginning of each REM sleep episode was identified by observation of ongoing polygraphic records (Fig. 1). From the room adjacent to the rat, the experimenter pressed a mechanical lever within 2-3 s of REM sleep onset, the animal's head was gently lifted and the animal was awakened. A detailed description and validation of this selective REM sleep deprivation method is described in an earlier publication (Datta et al., 2004). One of the important advantages of this REM sleep deprivation method is that it successfully eliminates $>75 \%$ of REM sleep without significantly reducing SWS.

Determination of behavioral states. For determining possible effects on sleep and wakefulness, polygraphic data were captured on-line in a computer using "Gamma" software (Grass product group, Astro-Med). From the captured data, three behavioral states were distinguished and scored visually using "Rodent Sleep Stager" software (Grass product group, Astro-Med). The three states were: Wakefulness (W), SWS, and REM sleep. The physiological criteria for the identification of these wake-sleep states were described in detail in earlier publications (Datta et al., 2002, 2004). In this study, the behavioral states of W, SWS, and REM sleep were scored in successive $5 \mathrm{~s}$ epochs. This epoch duration allowed us to quantify short periods of REM sleep (3-5 s) in rats in which REM sleep was selectively terminated (Datta et al., 2004).

Localized microinjections of RpCAMPS and vehicle control into the PPT. The microinjection system consisted of a 32-gauge stainless steel injector cannula with a 26-gauge collar that extended $2.0 \mathrm{~mm}$ beyond the implanted guide tube. The collar was connected to a $1.0 \mu$ l motor-driven Hamilton microsyringe with PE 20 tubing. While the animal was connected to the recording system, the stylettes were removed and an injector filled with either control vehicle ( $100 \mathrm{nl}$ volume of $0.9 \%$ saline) or one of the three concentrations of RpCAMPS $(0.55,1.1$, and $2.2 \mathrm{nmol} / 100 \mathrm{nl})$ was introduced through one of the bilateral guide tubes for the injection. This procedure was repeated in the other guide tube. One minute after the insertion of the injector cannulae, $100 \mathrm{nl}$ of control saline or one of the three concentrations of RpCAMPS was bilaterally microinjected over a 60 s period (Pump II Pico Plus, Harvard Apparatus). The injector cannulae were gently withdrawn 2 min after the injections, and the stylettes were reinserted into the guide tube. During the microinjections, animals were free to move around the cage with the cannulae in place. Each rat received a total of two microinjections ( $100 \mathrm{nl}$ each, one in the right and one in the left PPT) in a single experimental recording session. 
A

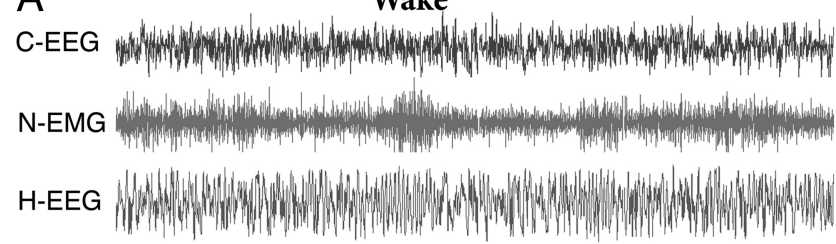

B

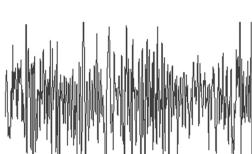

N-EMG

H-EEG

W.

C
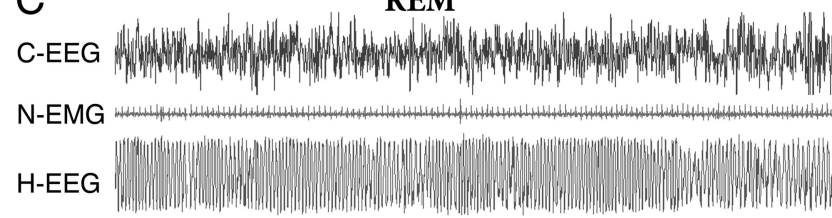

D
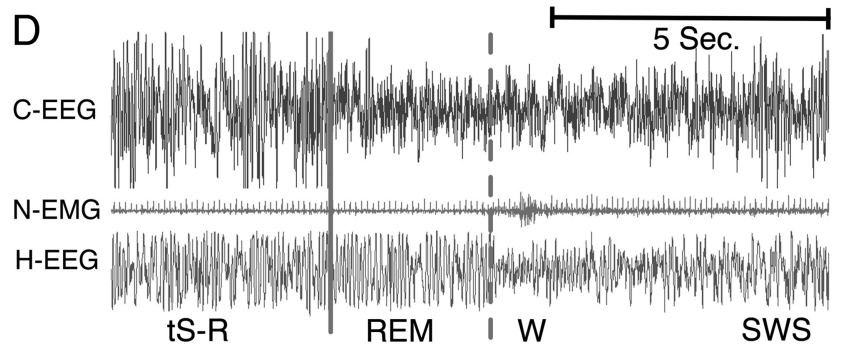

Figure 1. Polygraphic recordings of wake-sleep stages and the selective REM deprivation protocol. Representative polygraphic traces of the cortical electroencephalogram (C-EEG), neck muscle electromyogram (N-EMG), and hippocampal EEG (H-EEG) recorded simultaneously from a freely moving adult rat. The figure shows the polygraphic appearance of wakefulness $(\boldsymbol{A})$, slow-wave sleep $(\boldsymbol{B})$, and REM sleep $(\boldsymbol{C})$. D shows an example of a REM sleep episode that was prematurely terminated (within $3 \mathrm{~s}$ ) using our selective REM sleep deprivation protocol. In $\boldsymbol{D}$, the solid vertical line indicates the end of a tS-R (a transitional state between SWS and REM sleep) and the beginning of a REM sleep episode. The dashed vertical line marks the end of the prematurely terminated REM sleep episode. Note a brief period of $W$ at the end of this REM episode that ultimately changed to SWS, demonstrating that our selective REM sleep deprivation method did not increase the amount of time spent in W by increasing motor activity, and also did not decrease the amount of time spent in SWS. The bar indicates a $5 \mathrm{~s}$ time scale.

None of the rats were used for more than one microinjection recording session.

Recent microinjection studies have shown that a major advantage of the microinjection technique is its ability to stimulate a relatively circumscribed neuronal pool (Capece and Lydic, 1997; Datta et al., 2001, 2002). This view is supported by the biophysical studies of drug diffusion (Nicholson, 1985), and distribution kinetics (Herz and Teschemacher, 1971; Martin, 1991). Diffusion data obtained from radiolabeled drug studies showed that $30 \mathrm{~min}$ after brain microinjections of $500 \mathrm{nl}$ volumes, $72 \%$ of the drug remained within a radius of $0.75 \mathrm{~mm}$ (Yaksh and Rudy, 1978). In this study, the target area of the $100 \mathrm{nl}$ drug solutions injected into the PPT (approximate size: length: 1.0; height: 1.0; and width: 0.8 $\mathrm{mm}$ ), were well within the diffusion limit of this small volume. Moreover, in our recent microinjection mapping study in the PPT, it has been demonstrated that $100 \mathrm{nl}$ of glutamatergic, GABAergic, and intracellular signal transduction activators and inhibitors with drug concentrations similar to those used in this experiment, were ineffective when injections were $0.5 \mathrm{~mm}$ away from the center of the PPT (Datta et al., 2001; Datta, 2002, 2007; Ulloor et al., 2004). Additionally, using similar microinjection techniques, we demonstrated that ibotenic acid microinjection- induced cell loss in the brainstem of rats was limited to a maximum radius of $0.35 \mathrm{~mm}$ (Mavanji et al., 2004). Other sleep-wake related structures that are close to the PPT microinjection sites are $>1 \mathrm{~mm}$ away from the PPT microinjection site (Datta et al., 2003, 2009). Thus, it is highly improbable that the microinjected RpCAMPS used in this study (100 nl volume and $0.5-2.2 \mathrm{nmol}$ doses) diffused into the neighboring sleepwake areas in the brainstem. Therefore, we are confident that the pharmacological effects observed after microinjection of RpCAMPS significantly affected only the PPT.

Experimental design. On the day after the last adaptation recording session, when day-to-day variation in the total amount of REM sleep had stabilized, experimental recording sessions began. During the experimental recording session, each animal was connected to the polygraphic recording system at 8:55 A.M. In the next room, polygraphic signs of the sleep-wake cycle were continuously observed on a computer monitor (between 9:00 and 2:00 P.M.). The 40 rats were randomly divided into five groups. The experimental design of these studies is summarized in Figure $2 A$. Group 1 ( $n=8$ rats): received bilateral microinjections of vehicle control ( $0.9 \%$ saline, $100 \mathrm{nl}$ in the right and $100 \mathrm{nl}$ in the left PPT) at 12:00 P.M. while animals were recorded for $5 \mathrm{~h}$ session (between 9:00 A.M. and 2:00 P.M.) of undisturbed sleep-wakefulness (hereafter, group 1 is labeled as REM-Ctrl-REM; baseline REM sleep with vehicle control). The experimental protocol for group $2(n=8$ rats) was almost identical to the protocol described above for the REM-Ctrl-REM group, except that for group 2 animals, from 9:00 A.M. to 12:00 P.M., REM sleep episodes were selectively terminated at the beginning (within 3-5 s) of each episode while the animals were connected to the polygraphic recording system (hereafter, group 2 is labeled as "REM Dep-Ctrl-REM"; rebound REM sleep after selective REM sleep deprivation and application of vehicle control). The experimental protocol for group 3 ( $n=8$ rats) animals was similar to the protocol described above for the REM Dep-Ctrl-REM group, except that for group 3, animals were microinjected with a $0.55 \mathrm{nmol}$ (in a volume of $100 \mathrm{nl}$ ) dose of RpCAMPS (hereafter, group 3 is labeled as "REM Dep-RpCAMPS 0.55 nmol-REM"; selective REM sleep deprivation and unrestricted sleep-wakefulness after application of dose 1 of RpCAMPS). The experimental protocol for group 4 ( $n=8$ rats) animals was almost identical to the protocol described above for the REM Dep-RpCAMPS 0.55 nmol-REM group, except that for group 4, animals were microinjected with $1.1 \mathrm{nmol}$ dose of RpCAMPS (hereafter, group 4 is labeled as "REM Dep-RpCAMPS 1.1 nmol-REM"). The experimental protocol for group 5 ( $n=8$ rats) animals was similar to the protocol described above for the REM DepRpCAMPS 1.1 nmol-REM group, except that for group 5, animals were microinjected with $2.2 \mathrm{nmol}$ dose of RpCAMPS (hereafter, group 5 is labeled as "REM Dep-RpCAMPS 2.2 nmol-REM"). In addition to these 40 animals, four rats were subjected to a selective REM sleep deprivation protocol for the entire $5 \mathrm{~h}$ recording period (between 9:00 A.M. and 2:00 P.M.) and received bilateral microinjections of vehicle control at 12:00 P.M. (REM Dep-Ctrl-REM Dep group).

To study the mechanism of rebound REM sleep, rats were selectively deprived of REM sleep for a period of $3 \mathrm{~h}$, based on earlier studies that have demonstrated that $3 \mathrm{~h}$ of this type of REM sleep deprivation is sufficient to induce significant rebound REM sleep without increasing the total amount of time spent in W (Bandyopadhya et al., 2006; Shea et al., 2008). Additionally, using a $3 \mathrm{~h}$ period of REM sleep deprivation is advantageous because it does not reduce the total amount of time spent in SWS. However, the effects of RpCAMPS microinjection into the PPT were studied for only a $2 \mathrm{~h}$ period. The selection of this $2 \mathrm{~h}$ period was based on the fact that earlier studies have shown that after $3 \mathrm{~h}$ of selective REM sleep deprivation, the amount of REM sleep recovery is maximal during the second hour of the recovery sleep-wake period. Since the goal of this study was to identify the molecular mechanisms of REM sleep recovery, we recorded sleep-wake activity for $2 \mathrm{~h}$ and then immediately killed the rats to quantify the expression of PKA-CU and PKA activity in the PPT. To examine the effects on sleep-wake values, preinjection sleep-wake values of drug-treated groups were compared with the preinjection sleep-wake values of the vehicle control treated group. Similarly, postinjection sleep-wake values of drug-treated groups were compared with the postinjection sleep-wake values of the vehicle control 
treated group. To avoid confounded accounting, sleep-wake variables were not compared between $3 \mathrm{~h}$ of preinjection and $2 \mathrm{~h}$ of postinjection values.

Tissue collection. Immediately after the end of the experimental recording session (at 2:00 P.M.), rats were killed with carbon dioxide, and the brains were quickly removed and frozen using dry ice. To minimize possible variations due to differences in the sleep-wake state at the time of death, all animals were awakened by shaking their cages and kept awake for $1 \mathrm{~min}$ before they were killed. To rule out any diurnal factors contributing to the different levels of PKA activity and PKA-CU in the different groups, all rats were killed at a fixed time of day. The frozen brains were cut in the transverse plane in $300 \mu \mathrm{m}$ thick sections with a Vibratome (series 3000; Technical Products International). Under a dissecting microscope, the PPT was dissected on an ice-chilled Petri dish as described earlier (Ulloor and Datta, 2005; Bandyopadhya et al., 2006; Datta et al., 2008). The PPT tissue collection region was as follows: anteroposterior, between $7.7 \mathrm{~mm}$ and $8.7 \mathrm{~mm}$ posterior to the bregma; mediallateral, between $1.5 \mathrm{~mm}$ and $2.5 \mathrm{~mm}$ lateral to the midline. The dissected tissues from the PPT region, containing tissues from the both left and right side of the brain, of each individual rat were stored separately in prechilled microcentrifuge tubes at $-80^{\circ} \mathrm{C}$ until assayed.

Western blotting for the quantification of $P K A-C U$. The individual frozen tissue samples were rapidly sonicated (Sonic Dismembrator, Model 100, Fisher Scientific) in 5 volumes (w/v) of ice-cold mammalian CelLytic buffer (Sigma Biochemicals). The homogenates were then centrifuged at $10,000 \mathrm{rpm}$ for $10 \mathrm{~min}$ at $4^{\circ} \mathrm{C}$. The amount of protein in the supernatant (soluble fraction) of individual samples was then measured using Bradford's method (Bradford, 1976). Approximately $25 \mu \mathrm{g}$ of protein from each sample were heated (for $3 \mathrm{~min}$ at $95^{\circ} \mathrm{C}$ ) in Laemmli's $1 \times$ protein loading buffer (Laemmli, 1970). These denatured samples were resolved onto $12 \%$ SDS-polyacrylamide gels by standard electrophoresis. Separated proteins were then electro-transferred onto polyvinylidene difluoride (PVDF) membranes (Bio-Rad Laboratories). The membranes were probed for PKA-CU (anti-human $\mathrm{PKA}_{\mathrm{C} \alpha}$; clone 5B, BD-Biosciences; dilution 1:1500 dilution), and protein loading was controlled for by subsequent assessment of actin immunoreactivity (anti-actin; clone AC-40; Sigma Chemicals; 1:1500 dilution) on the same membrane. The resulting immunocomplexes were detected with a chemiluminescent substrate (SuperSignal, West Femto, Pierce). The quantification of the immunoreactive band was performed using a Kodak imaging densitometer (PerkinElmer). For the quantification of PKA-CU levels in the PPT, the net intensity of each PKA-CU chemiluminescent signal was first normalized with the net intensity of the corresponding actin chemiluminescent signal.

Estimation of PKA activity. For the PKA activity assay, the tissue homogenate was further purified by passing it through a mini spin column (Micro Bio-spin 6 chromatographic column, Bio-Rad). Before loading the extract, the column was prepared and equilibrated. Excess of the suspension solution present with the matrix was removed and $250 \mu \mathrm{l}$ of CelLytic buffer was added to equilibrate it. Any remaining amount of liquid that remained in the column bed after equilibration was also removed by spinning the column at $5000 \mathrm{rpm}$ for $30 \mathrm{~s}$ at $4^{\circ} \mathrm{C}$. The $200 \mu \mathrm{l}$ of
B

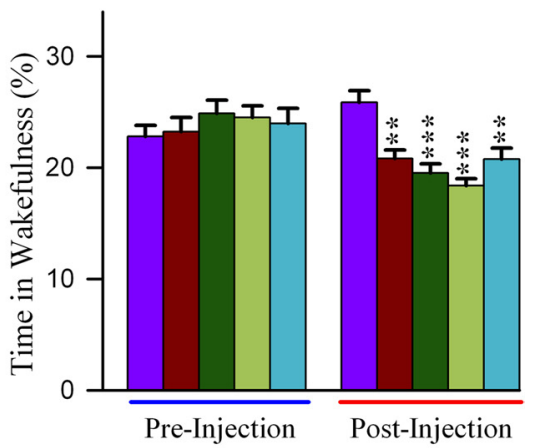

D
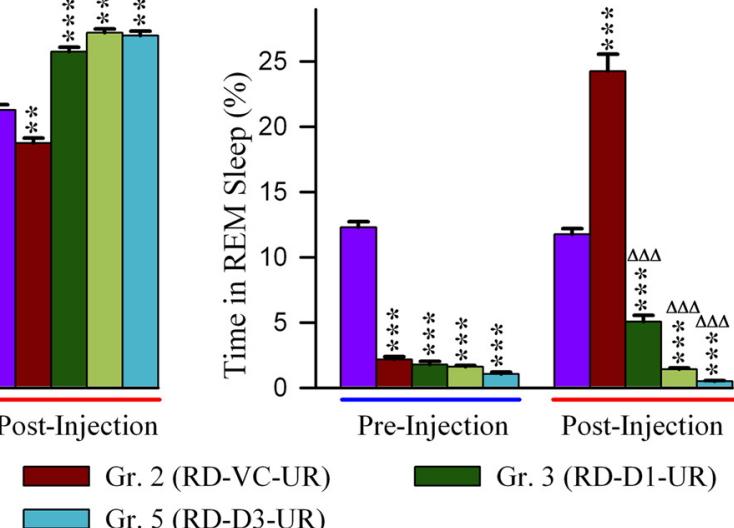

$\square$ Gr. 3 (RD-D1-UR)

Figure 2. Effects of selective REM sleep deprivation and RpCAMPS microinjections into the PPT on wake-sleep states. $\boldsymbol{A}$ Experimental design: Group 1 rats were allowed to have a $3 \mathrm{~h}$ period (9:00 A.M. to 12:00 P.M.) of undisturbed sleep-Wake (S-W)

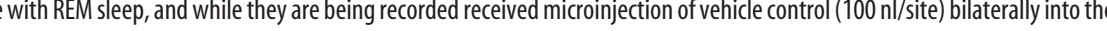
group 2 was identical to group 1, except REM sleep episodes in group 2 rats were selectively terminated from the ongoing $S-W$ (D) $0.55 \mathrm{nmol}$ of RpCAMPS (D1-Rp-CAMPS), group 4 received $1.1 \mathrm{nmol}$ of RpCAMPS (D2-Rp-CAMPS), and group 5

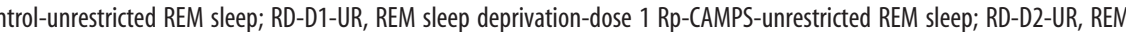
REM sleep. Post hoc tests (Bonferroni posttest): ${ }^{* *} p<0.01$ and ${ }^{* * *} p<0.001$, compared with group 1 (UR-VC-UR); $\Delta \Delta \Delta p<$ 0.001 , compared with group 2 (RD-VC-UR).

crude extract was then loaded on the equilibrated column and spun at $5000 \mathrm{rpm}$ for $2 \mathrm{~min}$ at $4^{\circ} \mathrm{C}$. Afterward, the flow-through solution was collected and saved as cleared extract. To prevent any loss of enzymatic activity in the preparation, a glycerol solution was added to the cleared extract creating a final concentration of $10 \%$. An aliquot of the glycerolmixed cleared extract was used to measure the protein concentration [by the Bradford method (Bradford, 1976)]. The remainder was saved at $-20^{\circ} \mathrm{C}$ until further used. In this study, PKA activity was measured using a nonradioactive assay method, the PepTag assay system developed by the Promega Corporation (White and Shultz, 1992; Sahir et al., 2001; Goueli et al., 2004). For the activity assay, empty $1.5 \mathrm{ml}$ Eppendorf tubes were placed on ice and appropriately labeled (one for each experimental sample, one for the positive control, and another for the negative control). Once chilled, a $5 \mu \mathrm{l}$ volume of PKA buffer (containing $100 \mathrm{~mm}$ Tris- $\mathrm{HCl}, \mathrm{pH}$ 7.4, $50 \mathrm{mM} \mathrm{MgCl}_{2}$, and $5 \mathrm{~mm} \mathrm{ATP}$ ) was poured in each tube, followed by $5 \mu \mathrm{l}$ volume of PKA substrate (containing $2 \mu \mathrm{g}$ of colored PepTag A1 Kemptide protein). Next, $1 \mu \mathrm{l}$ of company supplied protector solution was added to each tube containing PKA buffer and substrate. At this point, a $10 \mu \mathrm{l}$ volume of brain extract (containing $2 \mu \mathrm{g}$ of total protein) was added only to tubes labeled as experimental conditions. No brain extract was added to either the positive or negative control tube. 
Instead, $10 \mu \mathrm{l}$ of company supplied standard enzyme (containing $20 \mathrm{ng}$ of purified form of the PKA catalytic subunit) was added to the positive control tube, and $10 \mu \mathrm{l}$ of ice-cold deionized water was added to the negative control tube. After adding all the required reagents and the appropriate enzyme, the final volume of each tube was adjusted to a total volume of $25 \mu \mathrm{l}$ by adding $4 \mu \mathrm{l}$ of ice-cold deionized water. The reaction mixes were then incubated at room temperature for $60 \mathrm{~min}$. After incubation, the tubes were placed in a $95^{\circ} \mathrm{C}$ water bath for $10 \mathrm{~min}$ to stop the reaction. The samples were then quickly spun in a Microfuge to pull down the condensate and ultimately loaded in a $1 \%$ agarose gel. The gel used for the study was prepared in $50 \mathrm{~mm}$ Tris- $\mathrm{HCl}$ buffer, $\mathrm{pH}$ 8.0, and was electrophoresed (20-30 min at 100 constant volts) in the same solution. After electrophoresis, the gel was photographed under UV light using a Kodak Image-station (440CF). For quantification of the bands, which represented both the phosphorylated and nonphosphorylated Kemptides, the image intensities were converted to optical density (OD) using the Kodak Image analysis software program.

Statistical analysis. To determine the effects of selective REM sleep deprivation and intra PPT microinjections of RpCAMPS on changes in sleep-wake data, the polygraphic measures of the rats were analyzed to calculate the total percentages of time spent in W, SWS, REM sleep, and number of REM sleep episodes in two blocks: (1) the first $3 \mathrm{~h}$ of the experimental sleep-wake recordings (before intra PPT microinjections; between 9:00 A.M. and 12:00 P.M.), and (2) the last $2 \mathrm{~h}$ of the experimental sleep-wake recordings (after intra PPT microinjections; between 12:00 P.M. and 2:00 P.M.), before they were killed to collect brain tissues. The sleep-wake data were first subjected to two-way ANOVAs and post hoc Bonferroni tests to compare the total percentages of time spent in W, SWS, and REM sleep in the REM-Ctrl-REM, REM Dep-Ctrl-REM, REM Dep-RpCAMPS 0.55 nmol-REM, REM Dep-RpCAMPS 1.1 nmol-REM, and REM Dep-RpCAMPS $2.2 \mathrm{nmol}$-REM groups. To rule out the possibility that the baseline total percentages of time spent in W, SWS, REM sleep among the five groups of rats are different, the values in the final $5 \mathrm{~h}$ baseline recording session were subjected to one-way ANOVA. Onefactor ANOVAs and post hoc tests (Bonferroni's multiple-comparison test) were used to compare the levels of PKA-CU and PKA activity in the REM-Ctrl-REM, REM Dep-Ctrl-REM, REM Dep-RpCAMPS 0.55 nmol-REM, REM Dep-RpCAMPS 1.1 nmol-REM, and REM DepRpCAMPS 2.2 nmol-REM groups. To assess the relative correlations between the levels of PKA-CU expression or PKA activity, and the percentages of time spent in W, SWS, or REM sleep during the last $2 \mathrm{~h}$ of experimental recordings (between 12:00 P.M. and 2:00 P.M.; recovery REM sleep recording period), Pearsons correlations, which assume a Gaussian distribution, were calculated (regression analysis). As a standard rule of statistical analysis, before individual statistical tests, group data were subjected to normality testing, which confirmed that the data meets normality assumptions. All statistical analyses were performed using GraphPad Prism statistical software.

\section{Results \\ Effects of selective REM sleep deprivation and RpCAMPS microinjection into the PPT on wake-sleep states}

The experimental protocols are illustrated in Figure $2 \mathrm{~A}$. The total percentages of time spent in W, SWS, REM sleep, and the number of REM sleep episodes during the final $5 \mathrm{~h}$ baseline recording session (between 9:00 A.M. and 2:00 P.M.) were not significantly different (one-way ANOVA) among the REM-Ctrl-REM, REM Dep-Ctrl-REM, REM Dep-RpCAMPS 0.55 nmol-REM, REM Dep-RpCAMPS 1.1 nmol-REM, and REM Dep-RpCAMPS 2.2 nmol-REM groups of animals. Thus, in the final baseline recording conditions, the groups were initially equal in terms of time spent in W, SWS, and REM sleep. In the REM-Ctrl-REM group of rats, the last $2 \mathrm{~h}$ of experimental recording session was comparable (Student's $t$ tests) to the last $2 \mathrm{~h}$ of baseline recording session in terms of time spent in W (baseline vs experimental; $24.2 \pm$ $2.3 \%$ vs $22.8 \pm 1.1 \%)$, SWS $(63.8 \pm 2.0 \%$ vs $65.3 \pm 1.8 \%)$, REM sleep $(11.4 \pm 1.6 \%$ vs $12.3 \pm 1.3 \%)$, and number of REM sleep episodes $(9.6 \pm 1.2$ vs $10.1 \pm 1.4)$. These results suggest that, during the experimental recording session, the REM-Ctrl-REM group of rats spent normal amounts of time in wake-sleep states, so that this group of animals was used as control.

\section{Effects on wakefulness}

The changes in the percentages of time spent in $\mathrm{W}$ during selective REM sleep deprivation and during unrestricted sleep-wake after bilateral microinjections of vehicle control, and the different concentrations of RpCAMPS are summarized in Figure 2B. A two-way ANOVA did not reveal a significant effect of treatment $\left[F_{(4,70)}=0.26, p=0.90\right]$ for the total percentage of time spent in W. However, the two-way ANOVA indicated a significant effect of time $\left[F_{(1,70)}=83.58, p<0.001\right]$, and a treatment $\times$ time interaction $\left[F_{(4,70)}=13.05, p<0.001\right]$. To determine the effect of the selective REM sleep deprivation protocol during the deprivation period, the total percentage of time spent in $\mathrm{W}$ during the first $3 \mathrm{~h}$ period of each of the experimental recording sessions in the REM Dep-Ctrl-REM, REM Dep-RpCAMPS 0.55 nmol-REM, REM Dep-RpCAMPS 1.1 nmol-REM, and REM Dep-RpCAMPS 2.2 nmol-REM groups was compared (Bonferroni posttest) with the percentage in the REM-Ctrl-REM group. The results of post hoc analysis (Bonferroni) of the total percentages of time spent in $\mathrm{W}$ are presented in Figure 2B. The results indicate that the total percentage of time spent in $\mathrm{W}$ in the REM Dep-Ctrl-REM, REM DepRpCAMPS 0.55 nmol-REM, REM Dep-RpCAMPS $1.1 \mathrm{nmol}-$ REM, and REM Dep-RpCAMPS 2.2 nmol-REM groups was not significantly different from that in the REM-Ctrl-REM group (Fig. 2B). These results suggest that the selective REM sleep deprivation protocol did not affect the total percentage of time spent in W. To determine the effect of selective REM sleep deprivation during the recovery sleep-wake period, the total percentage of time spent in $\mathrm{W}$ during the postinjection $2 \mathrm{~h}$ period of the experimental recording sessions in the REM Dep-Ctrl-REM group was compared (Bonferroni) with the REM-Ctrl-REM group. This test revealed that the total percentage of $\mathrm{W}$ in the REM Dep-Ctrl-REM group was significantly $(\mathrm{df}=70, t=3.45$, $p<0.01$ ) lower than in the REM-Ctrl-REM group (Fig. $2 B$ ), indicating that the selective REM sleep deprivation group of rats spent less time in $\mathrm{W}$ during the recovery sleep-wake period. To determine the effect of RpCAMPS microinjections into the PPT on $\mathrm{W}$, the total percentage of time spent in $\mathrm{W}$ during the postinjection $2 \mathrm{~h}$ period of each of the experimental recording sessions in the REM Dep-RpCAMPS 0.55 nmol-REM, REM Dep-RpCAMPS 1.1 nmol-REM, and REM Dep-RpCAMPS 2.2 nmol-REM groups was compared with the percentage in the REM Dep-Ctrl-REM group. The results indicate that the total percentage of time spent in W in the REM Dep-RpCAMPS 0.55 nmol-REM, REM Dep-RpCAMPS 1.1 nmol-REM, and REM Dep-RpCAMPS 2.2 nmol-REM groups was not significantly different from that in the REM Dep-Ctrl-REM group (Fig. 2B). These results suggest that microinjections of RpCAMPS into the PPT after selective REM sleep deprivation did not change the total percentage of time spent in $\mathrm{W}$.

\section{Effects on SWS}

The changes in the percentages of time spent in SWS during selective REM sleep deprivation and during unrestricted sleep-wake after bilateral microinjections of vehicle control and the different RpCAMPS concentrations are summarized in Figure 2C. Two-way ANOVA indicated a significant main effect of treatment $\left[F_{(4,70)}=\right.$ 47.48, $p<0.001]$, and a treatment $\times$ time interaction $\left[F_{(4,70)}=\right.$ $62.13, p<0.001]$, but no time effect $\left[F_{(1,70)}=3.31, p=0.08\right]$. To 
determine the effect of the selective REM sleep deprivation protocol during the deprivation period, the total percentage of time spent in SWS during the first $3 \mathrm{~h}$ period of each of the experimental recording sessions in the REM Dep-Ctrl-REM, REM Dep-RpCAMPS 0.55 nmol-REM, REM Dep-RpCAMPS 1.1 nmol-REM, and REM DepRpCAMPS 2.2 nmol-REM groups was compared (Bonferroni) with the percentage in the REM-Ctrl-REM group. The results of post hoc analysis of the total percentages of time spent in SWS are presented in Figure 2C. Post hoc analysis (Bonferroni) indicated that the total percentages of time spent in SWS were significantly greater in the REM Dep-Ctrl-REM ( $\mathrm{df}=70, t=3.37, p<0.01$ ), REM DepRpCAMPS 0.55 nmol-REM ( $\mathrm{df}=70, t=3.24, p<0.01$ ), REM Dep-RpCAMPS $1.1 \mathrm{nmol}-\mathrm{REM}$ ( $\mathrm{df}=70, t=3.40, p<0.01)$, and REM Dep-RpCAMPS 2.2 nmol-REM ( $\mathrm{df}=70, t=3.65, p<0.01)$ groups compared with the REM-Ctrl-REM group (Fig. $2 C$ ). These results suggest that the selective REM sleep deprivation protocol increased the total percentage of time spent in SWS. To determine the effect of selective REM sleep deprivation during the recovery sleep-wake period, the total percentage of time spent in SWS during the postinjection $2 \mathrm{~h}$ period of the experimental recording sessions in the REM Dep-Ctrl-REM group was compared (Bonferroni) with that in the REM-Ctrl-REM group. This test revealed that the total percentage of SWS in the REM Dep-Ctrl-REM group was significantly ( $\mathrm{df}=70, t=3.72, p<0.01$ ) lower (Fig. $2 C$ ), indicating that during the recovery sleep-wake period the selective REM sleep deprivation group of rats spent less time in SWS. To identify any SWS effect of RpCAMPS microinjections into the PPT, the total percentage of time spent in SWS during the $2 \mathrm{~h}$ postinjection period of each of the experimental recording sessions in the REM Dep-RpCAMPS 0.55 nmol-REM, REM Dep-RpCAMPS $1.1 \mathrm{nmol}-\mathrm{REM}$, and REM Dep-RpCAMPS $2.2 \mathrm{nmol}$-REM groups was compared with that in the REM Dep-Ctrl-REM group. The results of these tests indicated that the total percentage of time spent in SWS was significantly higher in the REM Dep-RpCAMPS 0.55 nmol-REM ( $\mathrm{df}=70, t=$ 13.17, $p<0.001$ ), REM Dep-RpCAMPS $1.1 \mathrm{nmol-REM} \mathrm{(} \mathrm{df}=70$, $t=15.94, p<0.001$ ), and REM Dep-RpCAMPS 2.2 nmol-REM $(\mathrm{df}=70, t=15.53, p<0.001)$ groups (Fig. $2 C$ ). These results suggest that the microinjections of RpCAMPS into the PPT after selective REM sleep deprivation increased the total percentage of time spent in SWS.

\section{Effects on REM sleep}

The changes in the percentages of time spent in REM sleep during selective REM sleep deprivation and during unrestricted sleepwake after bilateral microinjections of vehicle control, and the different RpCAMPS concentrations are summarized in Figure $2 D$. Two-way ANOVA indicated a significant main effect of treatment $\left[F_{(4,70)}=268.8, p<0.001\right)$, time $\left[F_{(1,70)}=251.2, p<\right.$ $0.001)$, and a treatment $\times$ time interaction $\left[F_{(4,70)}=214.0, p<\right.$ $0.001]$. The results of post hoc analysis (Bonferroni) showed that the total percentages of time spent in REM sleep during the $3 \mathrm{~h}$ deprivation period were significantly lower in the REM DepCtrl-REM (82.42\% less, df $=70, t=14.41, p<0.001)$, REM Dep-RpCAMPS 0.55 nmol-REM (85.52\% less, $\mathrm{df}=70, t=14.96$, $p<0.001$ ), REM Dep-RpCAMPS 1.1 nmol-REM (86.98\% less, $\mathrm{df}=70, t=15.27, p<0.001)$, and REM Dep-RpCAMPS 2.2 nmol-REM (91.62\% less, $\mathrm{df}=70, t=16.02, p<0.001)$ groups compared with the REM-Ctrl-REM group (Fig. $2 D$ ). These results suggest that the selective REM sleep deprivation protocol eliminated $>80 \%$ of the total amount of REM sleep. To determine the effect of selective REM sleep deprivation during the recovery sleep-wake period, the total percentage of time spent in REM sleep during the $2 \mathrm{~h}$ postinjection period of the experimental recording sessions in the REM Dep-Ctrl-REM group was compared (Bonferroni) with that in the REM-Ctrl-REM group. This test revealed that, compared with the REM-Ctrl-REM group, the REM Dep-Ctrl-REM group of rats had a significantly higher percentage of REM sleep (106.38\% more, $\mathrm{df}=70, t=17.78, p<00.001$ ), indicating that during the recovery sleep-wake period after selective REM sleep deprivation the REM Dep-Ctrl-REM group of rats had spent almost double the amount of time in REM sleep (Fig. $2 D)$. To determine the effect of RpCAMPS microinjections into the PPT on recovery REM sleep, the total percentage of time spent in REM sleep during the postinjection $2 \mathrm{~h}$ period of the experimental recording sessions in the REM Dep-RpCAMPS 0.55 nmol-REM, REM Dep-RpCAMPS 1.1 nmol-REM, and REM DepRpCAMPS 2.2 nmol-REM groups were compared (Bonferroni) with the percentage in the REM Dep-Ctrl-REM group. The results indicated that the total percentage of time spent in REM sleep was significantly lower in the REM Dep-RpCAMPS 0.55 nmol-REM (79.13\% less than the REM-Ctrl-REM group, $\mathrm{df}=$ $70, t=13.17, p<0.001)$, REM Dep-RpCAMPS 1.1 nmol-REM (94.14\% less, $\mathrm{df}=70, t=15.94, p<0.001)$, and REM DepRpCAMPS 2.2 nmol-REM (97.94\% less, $\mathrm{df}=70, t=15.53, p<$ 0.001 ) groups (Fig. 2D).

Unlike the total percentages of time spent in REM sleep, compared (Bonferroni) to the REM-Ctrl-REM group $(6.8 \pm 1.6)$, the mean number of REM sleep episodes was significantly greater in the REM Dep-Ctrl-REM (12.3 \pm 1.2 ; df $=35, t=13.17, p<$ 0.001), REM Dep-RpCAMPS 0.55 nmol-REM $(13.8 \pm 1.7$; $\mathrm{df}=$ $35, t=13.41, p<0.001)$, REM Dep-RpCAMPS 1.1 nmol-REM $(14.1 \pm 1.1 ; \mathrm{df}=35, t=14.17, p<0.001)$, and REM DepRpCAMPS 2.2 nmol-REM (13.9 \pm 1.4 ; df $=35, t=13.48, p<$ 0.001 ) groups during the $3 \mathrm{~h}$ selective REM sleep deprivation period. These results indicated that the number of attempts of REM sleep entries progressively increased within the selective REM sleep deprivation period. During the postinjection recording period, the mean number of REM sleep episodes in the REM Dep-Ctrl-REM group remained significantly greater (12.2 \pm 1.0 ; $\mathrm{df}=35, t=3.1, p<0.05)$ than in the REM-Ctrl-REM group $(9.1 \pm 0.8)$. Contrary to the REM Dep-Ctrl-REM group, during the postinjection recording period, the mean number of REM sleep episodes was significantly lower in the REM DepRpCAMPS 0.55 nmol-REM $(5.5 \pm 1.2 ; \mathrm{df}=35, t=14.81, p<$ $0.001)$, REM Dep-RpCAMPS $1.1 \mathrm{nmol}-\mathrm{REM}$ (2.0 \pm 0.5; $\mathrm{df}=35$, $t=18.27, p<0.001$ ), and REM Dep-RpCAMPS 2.2 nmol-REM $(1.1 \pm 1.2 ; \mathrm{df}=35, t=24.88, p<0.001)$ groups. These results indicated that the homeostatic drive for the initiation of REM sleep was suppressed after microinjections of RpCAMPS into the PPT.

\section{Effects of RpCAMPS microinjections and sleep-wake changes on PKA-CU expression in the PPT}

Changes in the expression of PPT PKA-CU and its relationship to sleep-wake changes are summarized in Figure 3. One-way ANOVA on the levels of PKA-CU expression in the PPT revealed a significant effect of treatment $\left[F_{(4,35)}=423.0, p<0.001\right]$. The results of post hoc analysis (Bonferroni's multiple-comparison test) on the levels of PKA-CU expression are presented in Figure $3 B$. Compared with the REM-Ctrl-REM group, the levels of PKA-CU expression were significantly higher in the REM Dep-Ctrl-REM group $(37.86 \%$ higher, $\mathrm{df}=35, t=10.9, p<0.001)$, but significantly lower in the REM Dep-RpCAMPS 0.55 nmol-REM (57.16\% less, $\mathrm{df}=35, t=16.5, p<0.001)$, REM Dep-RpCAMPS $1.1 \mathrm{nmol}-\mathrm{REM}$ (70.26\% less, $\mathrm{df}=35, t=20.3, p<0.001)$, and REM DepRpCAMPS 2.2 nmol-REM (78.64\% less, $\mathrm{df}=35, t=22.7, p<$ 
A
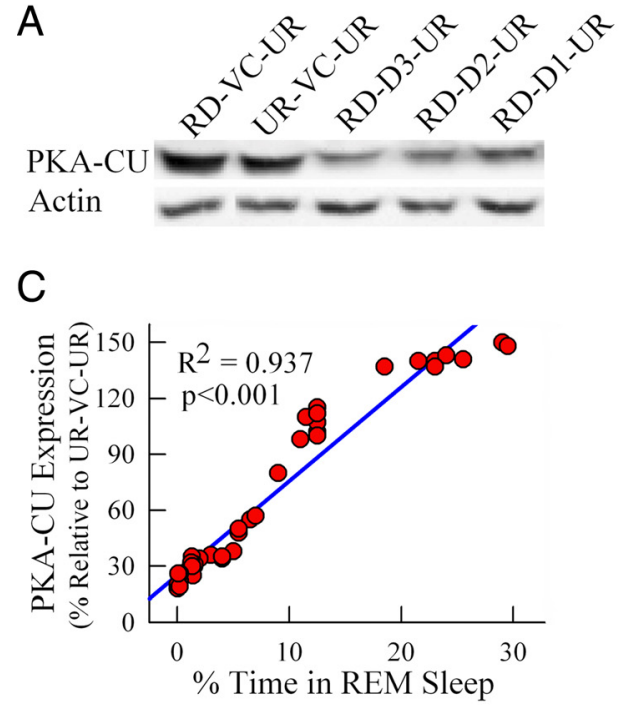

$\mathrm{D}$

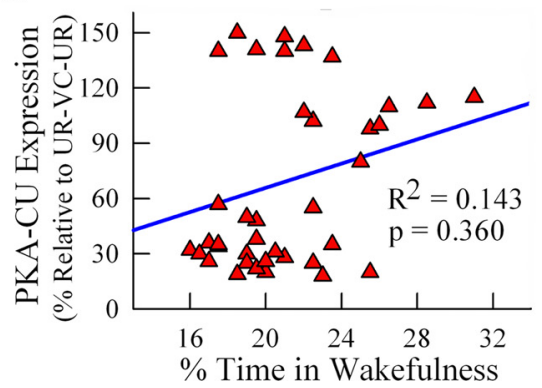

$\mathrm{B}$

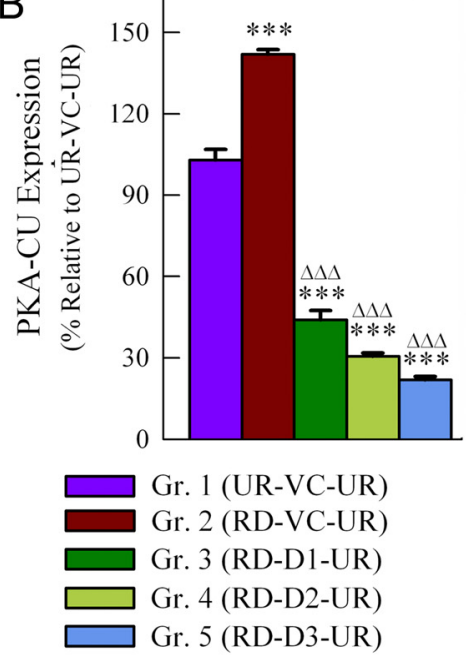

$\mathrm{E}$

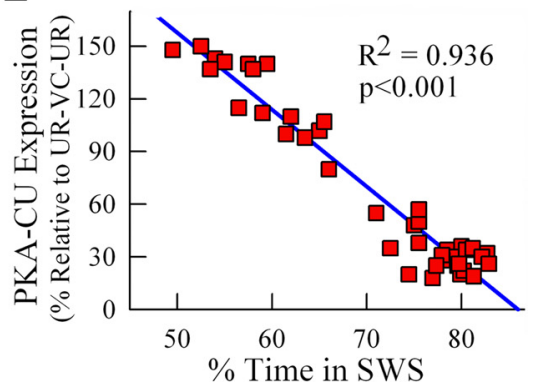

Figure 3. Relationship between recovery REM sleep and expression of PKA catalytic subunit protein (PKA-CU) in the PPT. $\boldsymbol{A}$, Representative Western blots of PKA-CU and actin in the PPT from UR-VC-UR, RD-VC-UR, RD-D1-UR, RD-D2-UR, and RD-D3-UR groups of animals (note that the lanes are random; the technician performing Western blotting and densitometric analysis was blind to experimental group). $\boldsymbol{B}$, Data from densitometric analysis of Western blots of PPT PKA-CU expressed as a percentage of control (Gr. 1, UR-VC-UR). Each bar represents the level of the PPT PKA-CU expression (mean \pm SE) of animals ( $n=8 /$ group) in group 1 (UR-VC-UR), group 2 (RD-VC-UR), group 3 (RD-D1-UR), group 4 (RD-D2-UR), and group 5 (RD-D3-UR). Note that $2 \mathrm{~h}$ after selective REM sleep deprivation and microinjections of vehicle control, PPT levels of PKA-CU expression increased (group 2). Also note that, with the microinjections of three different concentrations $(0.55,1.1$, and $2.2 \mathrm{nmol} / 100 \mathrm{nl})$ of Rp-CAMPS into the PPT in three different groups of animals (groups 3,4, and 5), PPT levels of PKA-CU expression decreased in a dose-dependent manner. Post hoc tests (Bonferroni's multiple-comparison test): ${ }^{* * *} p<0.001$, compared with group 1 (UR-VC-UR); $\Delta \Delta \Delta p<0.001$, compared with group 2 (RD-VC-UR). C $-E$, The level of PKA-CU expression in the PPT for each animal ( $n=40$ rats) is shown as a function of the percentage of time spent in REM sleep ( $\boldsymbol{C}$, wakefulness $(\boldsymbol{D})$, and SWS $(\boldsymbol{E})$ during the $2 \mathrm{~h}$ period (12:00 P.M. to 2:00 P.M.) of recovery sleep-wake $(S-W)$ cycle. Because some of the data points have identical values, not all 40 data points are visible in $\mathbf{C}-\boldsymbol{E}$. C, Plot of linear regression best fit (solid line; Pearson product-moment correlation) shows a statistically significant positive slope (Rsqr $=0.937 ; p<0.001)$. These data indicate that the level of PKA-CU expression in the PPT depends positively on the percentage of recovery REM sleep in the period of recovery $S-W$ cycle. $\boldsymbol{D}$, Plot of linear regression best fit did not reveal any significant relationship between the levels of PKA-CU expression in the PPT and total percentages of time spent in wakefulness $($ Rsqr $=0.143 ; p=0.360)$. These data indicate that the level of PKA-CU expression in the PPT did not vary as a function of wakefulness in the period of recovery $S-W$ cycle. $\boldsymbol{E}$, Plot of linear regression best fit revealed a significant negative relationship between the levels of PKA-CU expression in the PPT and total percentages of time spent in SWS (Rsqr $=0.936 ; p<0.001$ ). These data indicate that the level of PKA-CU expression in the PPT depends negatively on the percentage of SWS in the period of recovery S-W cycle. UR-VC-UR, unrestricted REM sleep-vehicle control-unrestricted REM sleep; RD-VC-UR, REM sleep deprivation-vehicle control-unrestricted REM sleep; RD-D1-UR, REM sleep deprivation-dose 1 Rp-CAMPS-unrestricted REM sleep; RD-D2-UR, REM sleep deprivation-dose 2 Rp-CAMPS-unrestricted REM sleep; and RD-D3-UR, REM sleep deprivation-dose 3 Rp-CAMPSunrestricted REM sleep. Post hoc tests (Bonferroni posttest): ${ }^{* *} p<0.01$ and ${ }^{* * *} p<0.001$, compared with group 1 (UR-VC-UR); $\Delta \Delta \Delta p<0.001$, compared with group 2 (RD-VC-UR).

0.001 ) groups (Fig. 3B). These results indicated that with increased and decreased total percentages of time spent in REM sleep, the expression of PKA-CU in the PPT increased and decreased, respectively. To determine the effects of RpCAMPS microinjections into the PPT on PKA-CU expression, the levels of PKA-CU expression in the REM Dep-RpCAMPS 0.55 nmol-REM, REM Dep-RpCAMPS 1.1 nmol-REM, and REM Dep-RpCAMPS
2.2 nmol-REM groups were also compared (Bonferroni's multiple-comparison test) with the levels in the REM Dep-Ctrl-REM group. The results of these comparisons showed that the expression of PKA-CU in the PPT was significantly lower in the REM Dep-RpCAMPS 0.55 nmol-REM (68.92\% less, $\mathrm{df}=35, t=27.4, p<0.001)$, REM Dep-RpCAMPS 1.1 nmol-REM (78.43\% less, $\mathrm{df}=35, t=31.2, p<0.001$ ), and REM Dep-RpCAMPS 2.2 nmol-REM (84.51\% less, $\mathrm{df}=35, t=33.6, p<0.001$ ) groups (Fig. 3B). These results indicated that the microinjections of RpCAMPS caused a dose-dependent reduction in PKA-CU expression in the PPT. Since the microinjections of RpCAMPS into the PPT decreased both REM sleep and expression of PKA-CU in the PPT, we expected to see a relationship (linear regression) between individual animals' total percentages of REM sleep and the level of PKA-CU expression in the PPT. The results of these regression analyses revealed a significant positive relationship with the total percentages of time spent in REM sleep $($ Rsqr $=0.937, F=568.32, p<0.001$; Fig. $3 C$ ), and a significant negative relationship with the total percentages of time spent in SWS $($ Rsqr $=0.936, F=555.67, p<0.001$; Fig. 3E). Similar regression analyses did not reveal any significant relationship between PKA-CU expression and the total percentages of time spent in $\mathrm{W}(\mathrm{Rsqr}=0.143, F=$ 6.366, $p=0.360$; Fig. 3D).

\section{Effects of RpCAMPS microinjections and sleep-wake changes on PKA activity in the PPT}

Changes in the level of PPT PKA activity and its relationship with sleep-wake changes are summarized in Figure 4. One-way ANOVA on the levels of PPT PKA activity revealed a significant effect of treatment $\left[F_{(4,35)}=487.8, p<0.001\right]$. The results of post hoc analysis (Bonferroni's multiplecomparison test) on the levels of PKA activity are presented in Figure 4B. Compared with the REM-Ctrl-REM group, the levels of PKA-CU expression were significantly higher in the REM Dep-Ctrl-REM group $(47.31 \%$ higher, $\mathrm{df}=35, t=13.23$, $p<0.001)$, but significantly lower in the REM Dep-RpCAMPS 0.55 nmol-REM (59.23\% less, $\mathrm{df}=35, t=16.59, p<$ 0.001), REM Dep-RpCAMPS 1.1 nmolREM $(73.92 \%$ less, $\mathrm{df}=35, t=20.71, p<0.001)$, and REM Dep-RpCAMPS 2.2 nmol-REM ( $83.47 \%$ less, $\mathrm{df}=35, t=23.38$, $p<0.001$ ) groups (Fig. $4 B$ ). These results indicated that PKA activity in the PPT increased and decreased with the increased and decreased total percentages of time spent in REM sleep, respectively. To determine the effects of RpCAMPS microinjections into the PPT on PKA activity, the levels of PKA activity in 
the REM Dep-RpCAMPS 0.55 nmolREM, REM Dep-RpCAMPS $1.1 \mathrm{nmol}$ REM, and REM Dep-RpCAMPS 2.2 nmol-REM groups were compared (Bonferroni's multiple-comparison test) with the REM Dep-Ctrl-REM group. The results of these comparisons showed that PKA activity in the PPT was significantly lower in the REM Dep-RpCAMPS 0.55 nmol-REM $(72.32 \%$ less, $\mathrm{df}=35, t=$ 29.82, $p<0.001)$, REM Dep-RpCAMPS 1.1 nmol-REM $(82.29 \%$ less, $\mathrm{df}=35, t=$ 33.94, $p<0.001$ ), and REM DepRpCAMPS 2.2 nmol-REM (88.78\% less, $\mathrm{df}=35, t=33.61, p<0.001)$ groups (Fig. $4 B)$. These results indicated that microinjections of RpCAMPS caused a dosedependent reduction of PKA activity in the PPT. Based on these results, we expected to see a relationship (linear regression analysis) between individual animals' PKA activity in the PPT and total percentages of time spent in REM sleep. The results of these analyses revealed a significant positive relationship with the total percentages of time spent in REM sleep $(\mathrm{Rsqr}=0.944, F=647.5, p<0.001$; Fig. $4 C$ ), and a significant negative relationship with the total percentages of time spent in SWS (Rsqr $=0.903, F=472.8$, $p<0.001$; Fig. $4 E$ ), but not with the total percentages of time spent in $\mathrm{W}$ (Rsqr $=$ 0.125, $F=$ 5.424, $p=0.373$; Fig. $4 D$ ). These results suggested that the amount of compensatory REM sleep after a selective REM sleep deprivation period depends on the level of PPT PKA activity. In addition to these subjects, four animals were subjected to selective REM sleep deprivation for the entire $5 \mathrm{~h}$ recording period. In these animals, during the last $2 \mathrm{~h}$, mean total percentage of REM sleep was only $1.8 \%$. The mean PKA activity of these animals (Fig. 4A, REM Dep-Ctrl-REM Dep) was $89.1 \%$ less than the mean PKA activity in the REM-Ctrl-REM group. Because PKA-CU expression is a positive indicator of PKA activation and the reduction of REM sleep decreased both PKA-CU expression and PKA activity in the PPT, one possibility is that the reduction in PKA activity may be linked to a reduction of PKA-CU expression. Linear regression analyses between individual animals' PKA activity and PKA-CU expression revealed a significantly positive relationship $(\mathrm{Rsqr}=0.945, F=$ 647.5, $p<0.001)$. These results suggested that, in the PPT, changes in PKA activity levels might have been caused by the changes in PKA-CU expression.

\section{Discussion}

The results presented here extend the role of the PPT in the regulation of REM sleep recovery following selective REM sleep
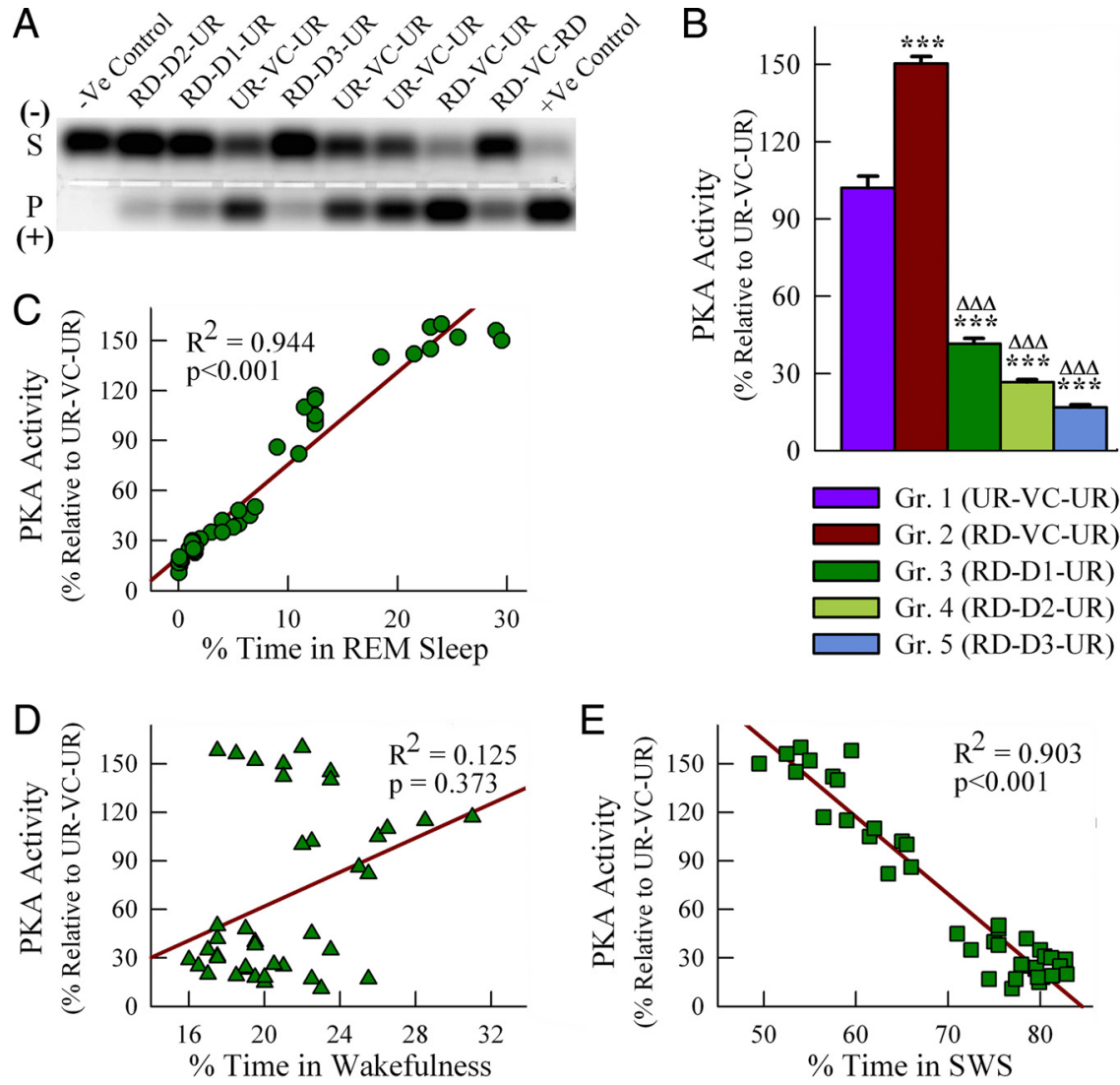

Figure 4. Relationship between recovery REM sleep and activation of PKA in the PPT. $A$, Representative agarose gel, visualized with UV light, showing the activity profile of the PKA in the PPT from UR-VC-UR, RD-VC-UR, RD-D1-UR, RD-D2-UR, RD-D3-UR, and RD-VC-RD groups of animals (note that the lanes are random; the technician performing Western blotting and densitometric analysis was blind to experimental group). The bottom band represents phosphorylated fluorescent-labeled Kemptide (P), and the top band represents the remaining unphosphorylated peptide (S). In the gel, phosphorylated product migrated toward the anode $(+)$, and unphosphorylated peptide migrated toward the cathode (-). - Ve Control, no protein or enzyme added; + Ve control, Purified catalytic subunit of PKA ( $20 \mathrm{ng}$ ); in each experimental sample $2 \mu \mathrm{g}$ of protein added. $\boldsymbol{B}$, Data from densitometric analysis of PPT PKA activity are expressed as a percentage of control (Gr. 1, UR-VC-UR). Each bar represents PPT PKA activity level (mean \pm SE) of the animals ( $n=8 /$ group) in group 1 (UR-VC-UR), group 2 (RD-VC-UR), group 3 (RD-D1-UR), group 4 (RD-D2-UR), and group 5 (RD-D3-UR). Note that $2 \mathrm{~h}$ after the selective REM sleep deprivation and microinjections of vehicle control, PPT PKA activity levels increased (group 2). Also note that after microinjections of three different concentrations $(0.55,1.1$, and $2.2 \mathrm{nmol} / 100 \mathrm{nl})$ of Rp-CAMPS into the PPT in three different groups of animals (groups 3, 4, and 5), PPT PKA activity levels decreased in a dosedependent manner. Post hoc tests (Bonferroni's multiple-comparison test): ${ }^{* * *} p<0.001$, compared with group 1 (UR-VC-UR); $\Delta \Delta \Delta p<0.001$, compared with group 2 (RD-VC-UR). C $-\boldsymbol{E}$, The level of PKA activity in the PPT for each animal ( $n=40$ rats) is shown as a function of the percentage of time spent in REM sleep $(\boldsymbol{C})$, wakefulness $(\boldsymbol{D})$, and SWS $(\boldsymbol{E})$ during the $2 \mathrm{~h}$ period (12:00 P.M. to 2:00 P.M.) of recovery sleep-wake ( $S-W$ cycle. Because some of the data points have identical values, not all 40 data points are visible in $\mathbf{C}-\boldsymbol{E}$. C, Plot of linear regression best fit (solid line; Pearsons product-moment correlation) shows a statistically significant positive slope (Rsqr $=0.944 ; p<0.001$ ). These data indicate that the level of PKA activity in the PPT depends positively on the percentage of recovery REM sleep in the period of recovery $S-W$ cycle. $D$, Plot of linear regression best fit did not reveal any significant relationship between the levels of PKA activity in the PPT and total percentages of time spent in wakefulness (Rsgr = $0.125 ; p=0.373$ ). These data indicate that the level of PKA activity in the PPT did not vary as a function of wakefulness in the period of recovery $S-W$ cycle. $E$, Plot of linear regression best fit revealed a significant negative relationship between levels of PKA activity in the PPT and total percentages of time spent in SWS (Rsqr $=0.903 ; p<0.001$ ). These data indicate that the level of PKA activity in the PPT depends negatively on the percentage of SWS in the period of recovery S-W cycle. UR-VC-UR, unrestricted REM sleep-vehicle control-unrestricted REM sleep; RD-VC-UR, REM sleep deprivation-vehicle control-unrestricted REM sleep; RD-D1UR, REM sleep deprivation-dose 1 Rp-CAMPS-unrestricted REM sleep; RD-D2-UR, REM sleep deprivation-dose 2 Rp-CAMPSunrestricted REM sleep; RD-D3-UR, REM sleep deprivation-dose 3 Rp-CAMPS-unrestricted REM sleep; RD-VC-RD, REM sleep deprivation-vehicle control-REM sleep deprivation.

deprivation. The principal findings of this study are as follows: (1) local application of the cAMP-PKA activation inhibitor, RpCAMPS, into the PPT blocked REM sleep recovery, and decreased the levels of PKA activity and PKA-CU expression in the PPT; (2) levels of PKA activity and PKA-CU expression in the PPT of individual animals were positively correlated with their 
total percentages of rebound REM sleep; (3) in the PPT, levels of PKA activity of individual animals were positively correlated with their levels of PKA-CU expression. These results provide direct evidence to suggest that the activation of the intracellular PKA signaling system in PPT cholinergic cells is an important molecular step for the expression of recovery REM sleep following REM sleep deprivation.

The results of this study demonstrate that this selective REM sleep deprivation protocol successfully reduced $\sim 85 \%$ of the total amount of REM sleep without increasing the total percentage of time spent in W. More importantly, unlike other REM sleep deprivation methods (Benington et al., 1994; Rechtschaffen et al., 1999; Deurveilher and Hennevin, 2001; Verret et al., 2005), this selective REM sleep deprivation protocol did not reduce the total percentage of time spent in SWS. During the selective REM sleep deprivation period, the number of interventions required to prevent REM sleep and total percentage of time spent in SWS increased. Additionally, during the recovery sleep period, the total percentage of REM sleep doubled in the animals that did not receive RpCAMPS in the PPT. Increased REM sleep propensity and amount of time spent in SWS during the period of REM sleep deprivation, and rebound REM sleep during the recovery sleep period, are physiological and behavioral indicators of activated homeostatic regulatory processes for REM sleep (Morden et al., 1967; Benington and Heller, 1994; Vivaldi et al., 1994; OcampoGarcés et al., 2000; Franken, 2002; Gvilia et al., 2006; Shea et al., 2008). Therefore, the results of this study demonstrate that this newly developed selective REM sleep deprivation protocol selectively activated the homeostatic drive for REM sleep.

The results of this study demonstrate that the application of a cAMP-PKA activation inhibitor, RpCAMPS, into the PPT blocked REM sleep recovery following selective REM sleep deprivation. In this study, cAMP-PKA activation inhibitor was microinjected into the part of the PPT (pars compacta) where most cells are known to be cholinergic (Mesulam et al., 1983; Jones and Beaudet, 1987; Rye et al., 1987; Vincent and Reiner, 1987; Datta et al., 2009). It is also known that the elimination of cholinergic cells in the PPT suppresses REM sleep deprivation-induced compensatory REM sleep (Deurveilher and Hennevin, 2001). The possibility that PPT cholinergic cells are involved in REM sleep deprivation-induced compensatory REM sleep is also suggested by other pharmacological studies that have shown systemic administration of cholinergic antagonists prevents REM sleep rebound after REM sleep deprivation (Salin-Pascual et al., 1991, 1992; Gillin et al., 1993; Stickgold et al., 1993). In addition, studies have shown that pharmacological agents that activate and inactivate PKA activity in the PPT also increase and decrease the expression of spontaneous REM sleep (Datta and Prutzman, 2005; Bandyopadhya et al., 2006; Datta, 2007). More recently, it has been demonstrated that $\sim 95 \%$ of the recovery REM sleep generating PPT neurons are cholinergic (Datta et al., 2009). Therefore, it is reasonable to suggest that the application of RpCAMPS into the PPT suppressed REM sleep recovery by affecting PPT cholinergic cells.

In this study, we measured PPT levels of PKA activity and PKA-CU expression. Analysis of PKA activity and PKA-CU expression in the PPT revealed that both PKA activity and PKA-CU expression levels increased with increased recovery REM sleep caused by REM sleep deprivation. Conversely, when animals were treated with RpCAMPS after selective REM sleep deprivation, PKA activity and PKA-CU expression levels in the PPT were reduced and recovery REM sleep was suppressed. Interestingly, PKA activity in the PPT was also decreased in animals that re- ceived only vehicle control microinjections into the PPT but were not allowed to have recovery REM sleep. These results suggest that the increase in recovery REM sleep is critical for increased levels of PKA activity and PKA-CU expression in the PPT. Additionally, the results of regression analysis revealed a strong positive relationship between the amount of recovery REM sleep and level of PKA activity in the PPT; in fact, $94.4 \%$ of the variance in homeostatic drive-induced REM sleep was explained by PKA activity $(\mathrm{Rsqr}=0.944)$. Similarly, PKA-CU expression in the PPT was a strong predictor of the amount of recovery REM sleep, explaining $93.7 \%$ of the variance $(\mathrm{Rsqr}=0.937)$. Furthermore, $94.5 \%$ of the variance in PKA-CU expression was explained by PKA activity in the PPT $($ Rsqr $=0.945)$. It is already known that increased activation of PKA increases PKA-CU levels in the cytoplasm (Lohmann and Walter, 1984; Taylor et al., 1990; Spaulding, 1993). A major nuclear target for free PKA-CU is CREB, which binds to CRE genes via the phosphorylation of CREB (Meinkoth et al., 1990). Phosphorylation of CREB then activates genes that ultimately produce a behavioral response specific to that particular cell. Indeed, it has been demonstrated that compensatory REM sleep increases phosphorylation of CREB in PPT cholinergic cells (Datta et al., 2009). Therefore, it is likely that the activation of PKA activity in PPT cholinergic cells increased transcription and translation processes by increasing PKA-CU expression, and that ultimately increased recovery REM sleep.

Understanding the role of the intracellular signal transduction pathway in a behaviorally active living organism is a vital step toward creating ways to converge functional biochemistry and integrative physiology. In this study, a selective inhibitor of cAMP-PKA activation, RpCAMPS, was microinjected into the PPT of freely moving animals to understand the mechanisms of homeostatic regulation of REM sleep. We must acknowledge that this approach has some interpretative limitations. For example, it is known that the PPT contains some noncholinergic cells (Kosaka et al., 1988; Clements and Grant, 1990; Lai et al., 1993; Ford et al., 1995; Liu et al., 1995; Wang and Morales, 2009), therefore, it is possible that those noncholinergic cells located within the targeted cholinergic cell groups will also be affected by the application of RpCAMPS. However, it has been shown that only $5 \%$ of the PPT cells that are pCREB-IR during recovery REM sleep following REM sleep deprivation, are noncholinergic (Datta et al., 2009). Therefore, it is highly unlikely that the robust behavioral and molecular effects observed in this study after microinjections of RpCAMPS into the PPT were caused by PPT noncholinergic cells. We also acknowledge that the RpCAMPS and vehicle control microinjected into the PPT may also diffuse to neighboring areas of the brainstem. However, it is unlikely that our PPT microinjection effects were influenced by neighboring sleep-wake related areas in the brainstem. This is also supported by the fact that, unlike the PPT, in the medial pontine reticular formation (mPRF) the expression of PKA-CU decreases with increased REM sleep (Bandyopadhya et al., 2006). This opposing effect is interesting because the mPRF is not only one of the anatomical sites closest to the PPT, but is also involved in the regulation of REM sleep (Shiromani and McGinty, 1986; Baghdoyan et al., 1987; Shiromani et al., 1992). It has also been shown that the microinjection of Sp-CAMPS (a drug that activates cAMP-PKA) into the MPRF prevents carbachol microinjection-induced REM sleep increase (Capece and Lydic, 1997). Another cholinergic region in the brainstem, close to the PPT, is the laterodorsal tegmental nucleus (LDT). Recently, it has been shown that increased or decreased REM sleep does not affect pCREB immunoreactivity in the LDT cholinergic cells (Datta et al., 2009). Therefore, based 
on the evidence discussed above, it appears that the activation of intracellular cAMP-PKA signaling for the generation of recovery REM sleep is an anatomically specific effect of PPT cholinergic cells. However, we acknowledge that this interpretation of anatomical specificity should be confirmed in future studies by microinjecting RpCAMPS in other anatomical sites that are known to be involved in the regulation of REM sleep.

In conclusion, molecular, pharmacological, and behavioral data reported here demonstrate that the activation of the cAMPdependent PKA signaling system in PPT cholinergic cells is an important cellular and molecular step for the generation of recovery REM sleep following selective REM sleep deprivation. These data are also consistent with the view that activation of PPT cholinergic cells is the primary cellular mechanism in the generation of spontaneous REM sleep. The current data provide a novel perspective on modulatory effects of PPT cholinergic cell activity in the homeostatic regulation of REM sleep.

\section{References}

Baghdoyan HA, Rodrigo-Angulo ML, McCarley RW, Hobson JA (1987) A neuroanatomical gradient in the pontine tegmentum for the cholinoceptive induction of desynchronized sleep signs. Brain Res 414:245-261.

Bandyopadhya RS, Datta S, Saha S (2006) Activation of pedunculopontine tegmental protein kinase A: a mechanism for rapid eye movement sleep generation in the freely moving rat. J Neurosci 26:8931-8942.

Benington JH, Heller HC (1994) REM-sleep timing is controlled homeostatically by accumulation of REM-sleep propensity in non-REM sleep. Am J Physiol 266:R1992-R2000.

Benington JH, Woudenberg MC, Heller HC (1994) REM-sleep propensity accumulates during 2-h REM-sleep deprivation in the rest period in rats. Neurosci Lett 180:76-80.

Bradford MM (1976) A rapid and sensitive methods for the quantification of microgram quantities of protein utilizing the principle of protein dye binding. Anal Biochem 72:248-254.

Capece ML, Lydic R (1997) cAMP and protein kinase A modulate cholinergic rapid eye movement sleep generation. Am J Physiol 273:R1430-R1440.

Clements JR, Grant S (1990) Glutamate-like immunoreactivity in neurons of the laterodorsal tegmental and pedunculopontine nuclei in the rat. Neurosci Lett 120:70-73.

Cook SA, Welch SP, Lichtman AH, Martin BR (1995) Evaluation of cAMP involvement in cannabinoid-induced antinociception. Life Sci 56:2049-2056.

Datta S (2000) Avoidance task training potentiates phasic pontine-wave density in the rat: a mechanism for sleep-dependent plasticity. J Neurosci 20:8607-8613.

Datta S (2002) Evidence that REM sleep is controlled by the activation of brainstem pedunculopontine tegmental kainate receptors. J Neurophysiol 87:1790-1798.

Datta S (2007) Activation of pedunculopontine tegmental PKA prevents GABA-B receptor activation-mediated rapid eye movement sleep suppression in the freely moving rat. J Neurophysiol 97:3841-3850.

Datta S, Maclean RR (2007) Neurobiological mechanisms for the regulation of mammalian sleep-wake behavior: Reinterpretation of historical evidence and inclusion of contemporary cellular and molecular evidence. Neurosci Biobehav Rev 31:775-824.

Datta S, Prutzman SL (2005) Novel role of brain stem pedunculopontine tegmental adenylyl cyclase in the regulation of spontaneous REM sleep in the freely moving rat. J Neurophysiol 94:1928-1937.

Datta S, Siwek DF (1997) Excitation of the brainstem pedunculopontine tegmentum cholinergic cells induces wakefulness and REM sleep. J Neurophysiol 77:2975-2988.

Datta S, Siwek DF (2002) Single cell activity patterns of pedunculopontine tegmentum neurons across the sleep-wake cycle in the freely moving rats. J Neurosci Res 70:611-621.

Datta S, Spoley EE, Patterson EH (2001) Microinjection of glutamate into the pedunculopontine tegmentum induces REM sleep and wakefulness in the rat. Am J Physiol 280:R752-R759.

Datta S, Spoley EE, Mavanji VK, Patterson EH (2002) A novel role of pe- dunculopontine tegmental kainate receptors: a mechanism of rapid eye movement sleep generation in the rat. Neuroscience 114:157-164.

Datta S, Mavanji V, Patterson EH, Ulloor J (2003) Regulation of rapid eye movement sleep in the freely moving rat: local microinjection of serotonin, norepinephrine, and adenosine into the brain stem. Sleep 26:513-520.

Datta S, Mavanji V, Ulloor J, Patterson EH (2004) Activation of phasic pontine-wave generator prevents rapid eye movement sleep deprivationinduced learning impairment in the rat: a mechanism for sleepdependent plasticity. J Neurosci 24:1416-1427.

Datta S, Li G, Auerbach S (2008) Activation of phasic pontine-wave generator in the rat: a mechanism for expression of plasticity-related genes and proteins in the dorsal hippocampus and amygdala. Eur J Neurosci 27:1876-1892.

Datta S, Siwek DF, Stack EC (2009) Identification of cholinergic and noncholinergic neurons in the pons expressing phosphorylated cyclic adenosine monophosphate response element-binding protein as a function of rapid eye movement sleep. Neuroscience 163:397-414.

Deurveilher S, Hennevin E (2001) Lesions of the pedunculopontine tegmental nucleus reduce paradoxical sleep (PS) propensity: evidence from a short-term PS deprivation study in rats. Eur J Neurosci 13:1963-1976.

El-Mansari M, Sakai K, Jouvet M (1989) Unitary characteristics of presumptive cholinergic tegmental neurons during the sleep-waking cycle in freely moving cats. Exp Brain Res 76:519-529.

Ford B, Holmes CJ, Mainville L, Jones BE (1995) GABAergic neurons in the rat pontomesencephalic tegmentum: codistribution with cholinergic and other tegmental neurons projecting to the posterior lateral hypothalamus. J Comp Neurol 363:177-196.

Franken P (2002) Long-term vs. short-term processes regulating REM sleep. J Sleep Res 11:17-28.

Garcia-Rill E (1991) The pedunculopontine nucleus. Prog Neurobiol 36:363-389.

Garcia-Rill E, Skinner RD, Miyazato H, Homma Y (2001) Pedunculopontine stimulation induces prolonged activation of pontine reticular neurons. Neuroscience 104:455-465.

Garcia-Rill E, Kobayashi T, Good C (2003) The developmental decrease in REM sleep. Thalamus Relat Sys 2:115-131.

Garcia-Rill E, Charlesworth A, Heister D, Ye M, Hayar A (2008) The developmental decrease in REM sleep: the role of transmitters and electrical coupling. Sleep 31:673-690.

Gillin JC, Salin-Pascual R, Velazquez-Moctezuma J, Shiromani P, Zoltoski R (1993) Cholinergic receptor subtypes and REM sleep in animals and normal controls. Prog Brain Res 98:379-387.

Goueli S, Larson B, Hsiao K, Worzella T, Gallagher A, Mathews E (2004) High-throughput kinase screening using a universal luminescent kinase assay. Cell Notes 10:20-23.

Gvilia I, Turner A, McGinty D, Szymusiak R (2006) Preoptic area neurons and the homeostatic regulation of rapid eye movement sleep. J Neurosci 26:3037-3044.

Herz A, Teschemacher H-J (1971) Activities and sites of antinociceptive action of morphine-like analgesics and kinetics of distribution following intravenous, intracerebral and intraventricular application. Adv Drug Delivery Res 6:79-119.

Jones BE (2004) Paradoxical REM sleep promoting and permitting neuronal networks. Arch Ital Biol 142:379-396.

Jones BE, Beaudet A (1987) Distribution of acetylcholine and catecholamine neurons in the cat brain stem studied by choline acetyltransferase and tyrosine hydroxylase immunohistochemistry. J Comp Neurol 261:15-32.

Kosaka T, Tauchi M, Dahl JL (1988) Cholinergic neurons containing GABA-like and/or glutamic acid decarboxylase-like immunoreactivities in various brain regions of the rat. Exp Brain Res 70:605-617.

Laemmli UK (1970) Cleavage of structural proteins during the assembly of the head of bacteriophage T4. Nature 227:680-685.

Lai YY, Clements JR, Siegel JM (1993) Glutamatergic and cholinergic projections to the pontine inhibitory area identified with horseradish peroxidase retrograde transport and immunohistochemistry. J Comp Neurol 336:321-330.

Liu RH, Fung SJ, Reddy VK, Barnes CD (1995) Localization of glutamatergic neurons in the dorsolateral pontine tegmentum projecting to the spinal cord of the cat with a proposed role of glutamate on lumbar motoneuron activity. Neuroscience 64:193-208. 
Lohmann SM, Walter U (1984) Regulation of the cellular and subcellular concentrations and distribution of nucleotide-dependent protein kinases. Adv Cyclic Nucleotide Protein Phosphoryl Res 18:63-102.

Lydic R, Baghdoyan HA (2008) Acetylcholine modulates sleep and wakefulness: a synaptic perspective. In: Neurochemistry of sleep and wakefulness (Monti JM, Pandi-Perumal SR, Sinton CM, eds), pp 109-143. Cambridge, UK: Cambridge UP.

Martin JH (1991) Autoradiographic estimation of the extent of reversible inactivation produced by microinjection of lidocaine and muscimol in the rat. Neurosci Lett 127:160-164.

Mavanji V, Ulloor J, Saha S, Datta S (2004) Neurotoxic lesions of phasic pontine-wave generator cells impair retention of two-way active avoidance memory. Sleep 27:1282-1292.

Meinkoth JL, Ji Y, Taylor SS, Feramisco JR (1990) Dynamics of the distribution of cyclic AMP-dependent protein kinase in living cells. Proc Natl Acad Sci U S A 87:9595-9599.

Mesulam MM, Mufson EJ, Wainer BH, Levey AI (1983) Central cholinergic pathways in the rat: an overview based on an alternative nomenclature (Ch1-Ch6). Neuroscience 10:1185-1201.

Morden B, Mitchell G, Dement W (1967) Selective REM sleep deprivation and compensation phenomena in the rat. Brain Res 5:339-349.

Nicholson C (1985) Diffusion of an injected volume of a substance in brain tissue with arbitrary volume fraction and tortuosity. Brain Res 333:325-329.

Ocampo-Garcés A, Molina E, Rodríguez A, Vivaldi EA (2000) Homeostasis of REM sleep after total and selective sleep deprivation in the rat. J Neurophysiol 84:2699-2702.

Pace-Schott EF, Hobson JA (2002) The neurobiology of sleep: genetics, cellular physiology and subcortical networks. Nat Rev Neurosci 3:591-605.

Paxinos G, Watson C (1997) The rat brain in stereotaxic coordinates. San Diego: Academic.

Punch LJ, Self DW, Nestler EJ, Taylor JR (1997) Opposite modulation of opiate withdrawal behaviors on microinjection of a protein kinase A inhibitor versus activator into the locus coeruleus or periaqueductal gray. J Neurosci 17:8520-8527.

Rechtschaffen A, Bergmann BM, Gilliland MA, Bauer K (1999) Effects of method, duration, and sleep stage on rebounds from sleep deprivation in the rat. Sleep 22:11-31.

Rye DB, Saper CB, Lee HJ, Wainer BH (1987) Pedunculopontine tegmental nucleus of the rat: cytoarchitecture, cytochemistry, and some extrapyramidal connections of the mesopontine tegmentum. J Comp Neurol 259:483-528.

Sahir N, Mas C, Bourgeois F, Simonneau M, Evrard P, Gressens P (2001) Caffeine-induced telencephalic vesicle evagination in early postimplantation mouse embryos involves cAMP-dependent protein kinase (PKA) inhibition. Cereb Cortex 11:343-349.

Salin-Pascual RJ, Granados-Fuentes D, Galicia-Polo L, Nieves E, Echeverry J (1991) Biperiden administration in normal sleep and after rapid eye movement sleep deprivation in healthy volunteers. Neuropsychopharmacology 5:97-102.

Salin-Pascual RJ, Jimenez-Anguiano A, Granados-Fuentes D, Drucker-Colin R (1992) Effects of biperiden on sleep at baseline and after $72 \mathrm{~h}$ of REM sleep deprivation in the cat. Psychopharmacology 106:540-542.

Schafe GE, LeDoux JE (2000) Memory consolidation of auditory Pavlion fear conditioning requires protein synthesis and protein kinase $\mathrm{A}$ in the amygdala. J Neurosci 20:RC96(1-5).

Self DW, Nestler EJ (1995) Molecular mechanisms of drug reinforcement and addiction. Annu Rev Neurosci 18:463-495.

Self DW, Genova LM, Hope BT, Barnhart WJ, Spencer JJ, Nestler EJ (1998) Involvement of cAMP-dependent protein kinase in the nucleus accumbens in cocaine self-administration and relapse of cocaine-seeking behavior. J Neurosci 18:1848-1859.

Shea JL, Mochizuki T, Sagvaag V, Aspevik T, Bjorkum AA, Datta S (2008) Rapid eye movement (REM) sleep homeostatic regulatory processes in the rat: changes in the sleep-wake stages and electroencephalographic power spectra. Brain Res 1213:48-56.

Shi WX, Bunney BS (1992) Roles of intracellular cAMP and protein kinase $\mathrm{A}$ in the actions of dopamine and neurotensin on midbrain dopamine neurons. J Neurosci 12:2433-2438.

Shiromani PJ, McGinty DJ (1986) Pontine neuronal response to local cholinergic infusion: relation to REM sleep. Brain Res 386:20-31.

Shiromani PJ, Kilduff TS, Bloom FE, McCarley RW (1992) Cholinergically induced REM sleep triggers Fos-like immunoreactivity in dorsol;ateral pontine regions associated with REM sleep. Brain Res 580:351-357.

Shouse MN, Siegel JM (1992) Pontine regulation of REM sleep components in cats: integrity of the pedunculopontine tegmentum (PPT) is important for phasic events but unnecessary for atonia during REM sleep. Brain Res 571:50-63.

Spaulding SW (1993) The ways in which hormones change cAMPdependent protein kinase subunits, and how such changes affect cell behavior. Endocr Rev 14:632-650.

Steriade M, Datta S, Paré D, Oakson G, Curró Dossi R (1990) Neuronal activities in brainstem cholinergic nuclei related to tonic activation processes in thalamocortical systems. J Neurosci 10:2541-2559.

Stickgold R, Williams J, Datta S, Quattrochi J, Hobson JA (1993) Suppression of eltoprazine-induced REM sleep rebound by scopolamine. Neuropharmacology 32:447-453.

Taylor SS, Buechler JA, Yonemoto W (1990) cAMP-dependent protein kinase: framework for a diverse family of regulatory enzymes. Annu Rev Biochem 59:971-1005.

Thakkar MM, Strecker RE, McCarley RW (1998) Behavioral state control through differential serotonergic inhibition in the mesopontine cholinergic nuclei: a simultaneous unit recording and microdialysis study. J Neurosci 18:5490-5497.

Ulloor J, Datta S (2005) Spatio-temporal activation of cyclic AMP response element-binding protein, activity-regulated cytoskeletal-associated protein and brain-derived nerve growth factor: a mechanism for pontinewave generator activation-dependent two-way active-avoidance memory processing in the rat. J Neurochem 95:418-428.

Ulloor J, Mavanji V, Saha S, Siwek DF, Datta S (2004) Spontaneous REM sleep is modulated by the activation of the pedunculopontine Tegmental GABA-B receptors in the freely moving rat. J Neurophysiol 91:1822-1831.

Van Hasstert PJM, Van Driel R, Jastorff B, Baraniak J, Stec WJ, De Wit RJW (1984) Competitive cAMP antagonists for cAMP-receptor proteins. J Biol Chem 259:10020-10024.

Verret L, Léger L, Fort P, Luppi PH (2005) Cholinergic and noncholinergic brainstem neurons expressing Fos after paradoxical (REM) sleep deprivation and recovery. Eur J Neurosci 21:2488-2504.

Vincent SR, Reiner PB (1987) The immunohistochemical localization of choline acetyltransferase in the cat brain. Brain Res Bull 18:371-415.

Vivaldi EA, Ocampo A, Wyneken U, Roncagliolo M, Zapata AM (1994) Short-term homeostasis of active sleep and the architecture of sleep in the rat. J Neurophysiol 72:1745-1755.

Wang HL, Morales M (2009) Pedunculopontine and laterodorsal tegmental nuclei contain distinct populations of cholinergic, glutamatergic and GABAergic neurons in the rat. Eur J Neurosci 29:340-358.

Wang LY, Salter MW, MacDonald JF (1991) Regulation of kainate receptors by cAMP-dependent protein kinase and phosphatases. Science 253:11321135 .

Webster HH, Jones BE (1988) Neurotoxic lesions of the dorsolateral pontomesencephalic tegmentum cholinergic cell area in the cat. II. Effects upon sleep-waking states. Brain Res 458:285-302.

White D, Shultz J (1992) A novel method for non-radioactive assays of specific protein kinases. Promega Notes 35:11.

Yaksh TL, Rudy TA (1978) Narcotic analgesics: CNS sites and mechanisms of action as revealed by intracerebral injection techniques. Pain 4:299359. 